\title{
Pseudo-goldstino and electroweak gauginos at the LHC
}

\author{
Ken-ichi Hikasa, ${ }^{a}$ Tao Liu, ${ }^{b}$ Lin Wang ${ }^{b}$ and Jin Min Yang ${ }^{c}$ \\ ${ }^{a}$ Department of Physics, Tohoku University, \\ Sendai 980-8578, Japan \\ ${ }^{b}$ Institut für Theoretische Teilchenphysik, Karlsruhe Institute of Technology (KIT), \\ D-76128 Karlsruhe, Germany \\ ${ }^{c}$ State Key Laboratory of Theoretical Physics, Institute of Theoretical Physics, Academia Sinica, \\ Beijing 100190, China \\ E-mail: hikasa@phys.tohoku.ac.jp, tao.liu2@kit.edu, wanglin@163.com, \\ jmyang@itp.ac.cn
}

ABSTRACT: The multi-sector SUSY breaking predicts the existence of pseudo-goldstino, which could couple more strongly to visible fields than ordinary gravitino. Then the lightest neutralino and chargino can decay into a pseudo-goldstino plus a $Z$-boson, Higgs boson or $W$-boson. In this note we perform a Monte Carlo simulation for the direct productions of the lightest neutralino and chargino followed by the decays to pseudo-goldstino. Considering scenarios with higgsino-like, bino-like or wino-like lightest neutralino, we find that the signal-to-background ratio at the high luminosity LHC is between 6 and $25 \%$ and the statistical significance can be above $5 \sigma$.

KeYWORDS: Supersymmetry Phenomenology

ARXiv EPRINT: 1403.5731 


\section{Contents}

1 Introduction 1

2 Theoretical review 2

3 Phenomenological study at LHC $\quad 6$

3.1 Higgsino-like LOSP $\left(|\mu|<M_{1}, M_{2}\right) \quad 7$

$\begin{array}{lll}3.2 & \text { Wino-like LOSP }\left(M_{2}<M_{1},|\mu|\right) & 10\end{array}$

3.3 Bino-like LOSP $\left(M_{1}<M_{2},|\mu|\right) \quad 12$

4 Conclusion $\quad 15$

\section{Introduction}

Supersymmetry (SUSY) remains the most popular theory for solving the hierarchy problem, albeit the recent discovery of a $125 \mathrm{GeV}$ Higgs boson, which makes most low energy SUSY models suffer from fine-tuning to some extent. ${ }^{1}$ From the viewpoint of model-building, the mechanism of SUSY breaking remains a puzzle. Usually, it is assumed that spontaneous breaking of SUSY occurs in some hidden sector and is mediated to visible fields by certain mechanism. Then a massless fermion named goldstino appears, which in the existence of local SUSY is absorbed into the longitudinal component of gravitino. If SUSY is broken in multiple sectors independently, each sector gives a goldstino $\eta_{i}$ with SUSY breaking scale $F_{i}$. One linear combination of $\eta_{i}$ is massless and eaten by the gravitino, while the orthogonal combination remains as a physical state and is named pseudo-goldstino. The property and related phenomenology of pseudo-goldstino have been investigated in the literature [8-22]. Comparing to the gravitino, the interactions of pseudo-goldstino are not totally constrained by the supercurrent and thus some of its coulpings could be large enough to have intriguing phenomenology. In the framework of gauge mediated SUSY breaking (GMSB), pseudogoldstino can make final states softer and more structured at colliders [20]. In GMSB with more than two hidden sectors the multi-photon signature was discussed in [21] and the LHC detectability for the Higgs boson decay into a pseudo-goldstino was examined in [22].

The non-observation of sparticles at the $7 \mathrm{TeV}$ and $8 \mathrm{TeV}$ runs of the $\mathrm{LHC}$ has set stringent bounds on colored sparticles. However, the electroweak sparticles are less constrained because of their small production rates, and can still have masses below $1 \mathrm{TeV}$. Theoretically, a light spectrum of electroweak sparticles is naturally predicted in some frameworks like anomaly mediation and non-minimal gauge mediations. So the study of electroweak sparticles, especially the light neutralinos and charginos, is rather important for testing SUSY at the LHC. At the LHC the neutralinos and charginos can be directly

\footnotetext{
${ }^{1}$ For the status of low energy SUSY models confronted with the LHC Higgs data, see, e.g., [1-7].
} 
produced through the Drell-Yan process and vector boson fusion. In many conventional scenarios with $R$-parity, the lightest neutralino is stable and just leads to missing energy in the experiments. But in some low scale gauge mediation scenarios the lightest neutralino can decay into a photon plus a gravitino. In the scenario of SUSY breaking in two hidden sectors, the lightest neutralino can decay to a pseudo-goldstino plus a $Z$-boson or Higgs boson. In this work we focus on such a two-sector SUSY breaking scenario to study the LHC detectability for the productions of lightest neutralino and chargino.

This work is organized as follows. In section II we will make a brief review on the framework with pseudo-goldstino and discuss its possible effect on the neutralino and chargino decays. Then in section III we take an effective way to study the corresponding signal at the LHC. Finally, we give our conclusions in section IV.

\section{Theoretical review}

Due to the non-renormalization theorem of superpotential, the spontaneous SUSY breaking is communicated to visible fields through the non-trivial Kähler potential $K$ and gauge kinetic function $f$. After integrating the hidden sector fields and parameterizing their information in a non-linear way [23-25]

$$
X_{i}=\frac{\eta_{i}^{2}}{2 F_{i}}+\sqrt{2} \theta \eta_{i}+\theta^{2} F_{i}
$$

the following representative term which contributes to the soft mass can be obtained

$$
\begin{aligned}
K & =\Phi^{\dagger} \Phi \sum_{i} \frac{m_{\phi, i}^{2}}{F_{i}^{2}} X_{i}^{\dagger} X_{i}, \\
f_{a b} & =\frac{1}{g_{a}^{2}} \delta_{a b}\left(1+\sum_{i} \frac{2 m_{a, i}}{F_{i}} X_{i}\right) .
\end{aligned}
$$

In the above equations, $\eta_{i}$ is the so-called goldstino and $m_{\phi, a}$ are respectively the soft masses for the chiral fields and gauginos. The trilinear $A$ terms and bilinear $B_{\mu}$ could also be constructed easily and we do not list them for simplicity. In the two-hiddensector scenario with the definition $F=\sqrt{F_{1}^{2}+F_{2}^{2}}$ and $\tan \theta=F_{2} / F_{1}$, the combination $G=\eta_{1} \cos \theta+\eta_{2} \sin \theta$ is eaten by the super-Higgs mechanism, while one pseudo-goldstino $G^{\prime}=-\eta_{1} \sin \theta+\eta_{2} \cos \theta$ is left. After substituting the expression of $X_{i}$ and making some rotations, we get the interaction Lagrangian up to order $1 / F_{i}$ :

$$
\begin{aligned}
\mathcal{L}_{G} & =\frac{m_{\phi}^{2}}{F} G \psi \phi^{*}-\frac{i m_{a}}{\sqrt{2} F} G \sigma^{\mu \nu} \lambda^{a} F_{\mu \nu}^{a}+\frac{m_{a}}{F} G \lambda^{a} D^{a}, \\
\mathcal{L}_{G^{\prime}} & =\frac{\widetilde{m}_{\phi}^{2}}{F} G^{\prime} \psi \phi^{*}-\frac{i \widetilde{m}_{a}}{\sqrt{2} F} G^{\prime} \sigma^{\mu \nu} \lambda^{a} F_{\mu \nu}^{a}+\frac{\widetilde{m}_{a}}{F} G^{\prime} \lambda^{a} D^{a} .
\end{aligned}
$$

Here the parameters $m$ and $\widetilde{m}$ are defined as

$$
\begin{array}{ll}
m_{a}=m_{a, 1}+m_{a, 2}, & \widetilde{m}_{a}=-m_{a, 1} \tan \theta+m_{a, 2} \cot \theta, \\
m_{\phi}^{2}=m_{\phi, 1}^{2}+m_{\phi, 2}^{2}, & \widetilde{m}_{\phi}^{2}=-m_{\phi, 1}^{2} \tan \theta+m_{\phi, 2}^{2} \cot \theta .
\end{array}
$$


In our analysis we assume a large hierarchy between $F_{1}$ and $F_{2}$ (we assume $F_{1} \gg F_{2}$ so that $\cot \theta$ is very large). In this case the pseudo-goldstino can couple more strongly to visible fields than ordinary goldstino (gravitino). Further, we will consider a small $\tilde{m}_{a}$ which happens for a large $\cot \theta\left(m_{a, 1} \tan \theta\right.$ is suppressed) and a very small $m_{a, 2}$ (such a tiny gaugino mass is easily achieved if the SUSY breaking sector $F_{2}$ approximately preserves R-symmetry [26]). In this special case, the pseudo-goldstino couplings with the photon or transverse $Z$-boson, which are proportional to $\tilde{m}_{a}$ in eq. (2.5), are suppressed. So in our following analysis we neglect the pseudo-goldstino couplings with the photon or transverse $Z$-boson.

Of course, a pseudo-goldstino should have a mass. At tree level its mass comes from the intrinsic property of SUGRA. Also it can get loop corrections, which are very modeldependent. In our analysis we assume that the pseudo-goldstino is rather light so that a neutralino can decay into a pseudo-goldstino plus a $Z$-boson. So our numerical results are only applicable to a rather light pseudo-goldstino (for a rather light pseudo-goldstino, say below $10 \mathrm{GeV}$, we can approximately neglect its mass in numerical calculations). The phenomenology of a rather massive pseudo-goldstino was considered in [20].

Now we look at the effects of pseudo-goldstino in concrete models. In the minimal supersymmetric standard model (MSSM), the Lagrangian for the neutralinos and charginos is given by

$$
\mathcal{L}=-\frac{1}{2} Y_{i j} \chi_{i} \chi_{j} h^{0}+G_{i j} \chi_{i}^{\dagger} \bar{\sigma}^{\mu} \chi_{j} Z_{\mu}+\left(I_{i j} \chi_{i}^{\dagger} \bar{\sigma}^{\mu} \chi_{j}^{+}+L_{i j} \chi_{j}^{-\dagger} \bar{\sigma}^{\mu} \chi_{i}\right) W_{\mu}^{-}+\text {h.c. } .
$$

Here $\chi_{i, j}$ represent the four neutralinos in the gauge eigenbasis $\left\{\tilde{B}, \widetilde{W}^{0}, \tilde{H}_{d}^{0}, \tilde{H}_{u}^{0}\right\}$ and their mass matrix is given by

$$
M_{\tilde{N}}=\left(\begin{array}{cccc}
M_{1} & 0 & -c_{\beta} s_{W} m_{Z} & s_{\beta} s_{W} m_{Z} \\
0 & M_{2} & c_{\beta} c_{W} m_{Z} & -s_{\beta} c_{W} m_{Z} \\
-c_{\beta} s_{W} m_{Z} & c_{\beta} c_{W} m_{Z} & 0 & -\mu \\
s_{\beta} s_{W} m_{Z} & -s_{\beta} c_{W} m_{Z} & -\mu & 0
\end{array}\right) .
$$

$\chi_{i, j}^{ \pm}$are charginos in the gauge eigenbasis $\left\{\widetilde{W}^{+}, \tilde{H}_{u}^{+}, \widetilde{W}^{-}, \tilde{H}_{d}^{-}\right\}$and their mass matrix is given by

$$
M_{\tilde{C}}=\left(\begin{array}{cc}
0 & X^{T} \\
X & 0
\end{array}\right), \quad X=\left(\begin{array}{cc}
M_{2} & \sqrt{2} s_{\beta} m_{W} \\
\sqrt{2} c_{\beta} m_{W} & \mu
\end{array}\right) .
$$

The couplings to the physical Higgs and gauge bosons are given by

$$
\begin{aligned}
& Y=\frac{1}{2}\left(\begin{array}{cccc}
0 & 0 & g^{\prime} s_{\alpha} & g^{\prime} c_{\alpha} \\
0 & 0 & -g s_{\alpha} & -g c_{\alpha} \\
g^{\prime} s_{\alpha}-g s_{\alpha} & 0 & 0 \\
g^{\prime} c_{\alpha}-g c_{\alpha} & 0 & 0
\end{array}\right), \quad G=\frac{g}{2 c_{W}}\left(\begin{array}{cccc}
0 & 0 & 0 & 0 \\
0 & 0 & 0 & 0 \\
0 & 0 & 1 & 0 \\
0 & 0 & 0 & -1
\end{array}\right) \text {, } \\
& I=g\left(\begin{array}{cccc}
0 & 0 & 0 & 0 \\
-1 & 0 & 0 & 0 \\
0 & 0 & 0 & 0 \\
0 & \frac{1}{\sqrt{2}} & 0 & 0
\end{array}\right), \quad L=g\left(\begin{array}{cccc}
0 & 0 & 0 & 0 \\
0 & 0 & 1 & 0 \\
0 & 0 & 0 & \frac{1}{\sqrt{2}} \\
0 & 0 & 0 & 0
\end{array}\right)
\end{aligned}
$$




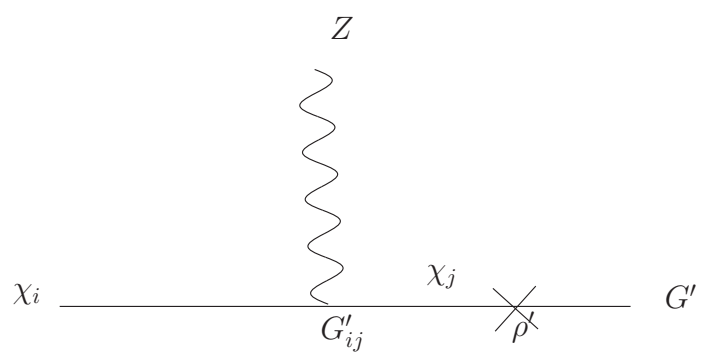

Figure 1. A diagrammatic show of interactions between $Z$-boson and pseudo-goldstino.

Since the contribution in eq. (2.5) is proportional to $\widetilde{m}_{\phi}^{2} / F$, there are two pseudo-goldstino interaction terms which should be added to the above Lagrangian:

$$
y_{i} G^{\prime} \chi_{i} h^{0}+\rho_{i} G^{\prime} \chi_{i}
$$

with the parameters $y_{i}$ and $\rho_{i}$ given by

$$
y=\frac{1}{\sqrt{2} F}\left(\begin{array}{c}
0 \\
0 \\
\widetilde{B}_{\mu} c_{\alpha}-\widetilde{m}_{H_{d}}^{2} s_{\alpha} \\
\widetilde{m}_{H_{u}}^{2} c_{\alpha}-\widetilde{B}_{\mu} s_{\alpha}
\end{array}\right), \quad \rho=\frac{v}{\sqrt{2} F}\left(\begin{array}{c}
0 \\
0 \\
\widetilde{m}_{H_{d}}^{2} c_{\beta}+\widetilde{B}_{\mu} s_{\beta} \\
\widetilde{m}_{H_{u}}^{2} s_{\beta}+\widetilde{B}_{\mu} c_{\beta}
\end{array}\right) .
$$

In the above matrices, $\alpha$ and $\beta$ are the mixing angles in the Higgs sector with $\tan \beta=$ $\left\langle H_{u}^{0}\right\rangle /\left\langle H_{d}^{0}\right\rangle$. We used the notations $s_{W}=\sin \theta_{W}, c_{W}=\cos \theta_{W}\left(\theta_{W}\right.$ is the Weinberg angle) and $s_{\beta}=\sin \beta, c_{\beta}=\cos \beta$.

The linear terms induce a small mixing between neutralinos and pseudo-goldstino, so we have to make a rotation to the mass eigenstate basis for neutralinos and then the small mass mixing can be treated perturbatively. For example, the vertex between $Z$-boson and pseudo-goldstino $G^{\prime}$ appears after a mass insertion $\rho^{\prime}$, as shown in figure 1 . The matrices $\rho_{i}^{\prime}$ and $G_{i j}^{\prime}$ are defined as

$$
\rho_{i}^{\prime}=\rho_{j} N_{j i}, \quad G_{i j}^{\prime}=G_{\ell m} N_{\ell i} N_{m j}
$$

where $N$ is the rotation to diagonalize the neutralino mass matrix. Other interactions could be obtained in the same way, such as the interaction between chargino and pseudogoldstino. Since in this scenario the couplings of pseudo-goldstino with photon or transverse component of $Z$-boson are negligible, the two possible decay channels for the lightest neutralino are $Z$ or $h$ plus $G^{\prime}$.

From the above analysis we can get the structure of the interactions for pseudogoldstino. However, there are many parameters involved, especially in the chargino and neutralino rotation matrices. So we only pick out some representative interactions to study the corresponding phenomenology.

To study the phenomenology, we employ the effective Lagrangian

$$
\mathcal{L}_{\text {eff }}=\frac{\widetilde{m}_{\phi}^{2}}{F}\left[g_{h \chi} h \chi^{0} G^{\prime}+g_{\chi Z} \bar{G}^{\prime} \bar{\sigma}^{\mu} \chi^{0} Z_{\mu}+g_{\chi W_{1}} \bar{G}^{\prime} \bar{\sigma}^{\mu} \chi^{+} W_{\mu}^{-}+g_{\chi W_{2}} \bar{G}^{\prime} \bar{\sigma}^{\mu} \chi^{-} W_{\mu}^{+}+\text {h.c. }\right] .
$$


Here we list all possible couplings, some of which may be turned off in specific cases. The decay widths of the lightest neutralino and chargino to pseudo-goldstino are given by

$$
\begin{aligned}
\Gamma\left(\chi^{0} \rightarrow h G^{\prime}\right) & =\frac{m_{\chi}}{16 \pi} \frac{g_{h \chi}^{2}}{F^{2}}\left(1-\frac{m_{h}^{2}}{m_{\chi}^{2}}\right)^{2}, \\
\Gamma\left(\chi^{0} \rightarrow Z G^{\prime}\right) & =\frac{m_{Z}^{2}}{8 \pi m_{\chi}} \frac{g_{\chi Z}^{2}}{F^{2}}\left(1-\frac{m_{Z}^{2}}{m_{\chi}^{2}}\right)\left[\frac{m_{\chi}^{4}}{2 m_{Z}^{4}}+\frac{m_{\chi}^{2}}{2 m_{Z}^{2}}-1\right], \\
\Gamma\left(\chi^{ \pm} \rightarrow W^{ \pm} G^{\prime}\right) & =\frac{m_{W}^{2}}{16 \pi m_{\chi}} \frac{\left(g_{\chi W_{1}}^{2}+g_{\chi W_{2}}^{2}\right) \widetilde{m}_{\phi}^{4}}{F^{2}}\left(1-\frac{m_{W}^{2}}{m_{\chi}^{2}}\right)\left[\frac{m_{\chi}^{4}}{2 m_{W}^{4}}+\frac{m_{\chi}^{2}}{2 m_{W}^{2}}-1\right] .
\end{aligned}
$$

The first two decay modes have been considered in [14] and we checked that our results agree with theirs. In our calculation we fix $\widetilde{m}_{\phi} / \sqrt{F}=0.1$ and all the couplings $g_{X}$ to be unity. Under these assumptions, the weak scale neutralino or chargino have the decay width at the order of $\sim 10^{-4} \mathrm{GeV}$ and the decay length $\Gamma^{-1} \sqrt{\left(E^{2}-m_{\chi}^{2}\right) / m_{\chi}^{2}} \sim 10^{-10} \mathrm{~cm}$ so they will decay inside the detector. Note that these parameters have no effects on the production rates of neutralino or chargino. As long as the neutralino and chargino only decay to pseudo-goldstino, their signal rates are not sensitive to these parameters.

About the parameter space in the neutralino/chargino sector, following the analysis in [27], we classify it according to the relative values of $M_{1,2}$ and $\mu$ : (i) $|\mu|<M_{1}, M_{2}$; (ii) $M_{2}<M_{1},|\mu|$; (iii) $M_{1}<M_{2},|\mu|$. Each case corresponds to a different property of the lightest neutralino, called the lightest ordinary sparticle (LOSP). In the first case, the LOSP is higgsino-like, which can not only decay to Higgs, but also decay to $Z$-boson though a mass insertion of $\rho$. In the second and third cases the LOSP is respectively wino-like and bino-like, which only decays to a Higgs boson plus a goldstino through its mass mixing with the higgsino. For the lightest chargino, which is too light to decay into a neutralino plus an on-shell $W$-boson, it now can decay into a $W$-boson plus a pseudo-goldstino. Note that in the second case the interaction vertex needs more than one insertion, so wino may mainly decay to gravitino. Since the decay to gravitino has the same collider signature, we assume the lightest chargino totally decay to pseudo-goldstino.

Note that in addition to the above decays, the neutralino can also decay to a real goldstino (gravitino), which may be competitive and need to be checked. The corresponding decay widths are given by $[28,29]$

$$
\begin{aligned}
& \Gamma\left(\chi^{0} \rightarrow \gamma G\right)=\left|N_{11} c_{W}+N_{12} s_{W}\right|^{2} \frac{m_{\chi}^{5}}{16 \pi F^{2}} \\
& \Gamma\left(\chi^{0} \rightarrow Z G\right)=\left(\left|N_{11} s_{W}-N_{12} c_{W}\right|^{2}+\frac{1}{2}\left|N_{13} c_{\beta}-N_{14} s_{\beta}\right|^{2}\right)\left(1-\frac{m_{Z}^{2}}{m_{\chi}^{2}}\right)^{4} \frac{m_{\chi}^{5}}{16 \pi F^{2}} .
\end{aligned}
$$

Here we see that the decay $\chi^{0} \rightarrow \gamma G$ is suppressed for a higgsino-like neutralino. So in the following we demonstrate the results for a bino-like neutralino and compare with the decays into a pseudo-goldstino. For numerical calculations, we fix the parameters $\tan \beta=10$, $M_{1}=200 \mathrm{GeV}, M_{2}=500 \mathrm{GeV}$ and $\mu=1.0 \mathrm{TeV}$. The soft mass $\tilde{m}_{\phi}$ is a combination of Higgs soft parameters whose values can be obtained from SOFTSUSY [30] once $\tan \beta$, 


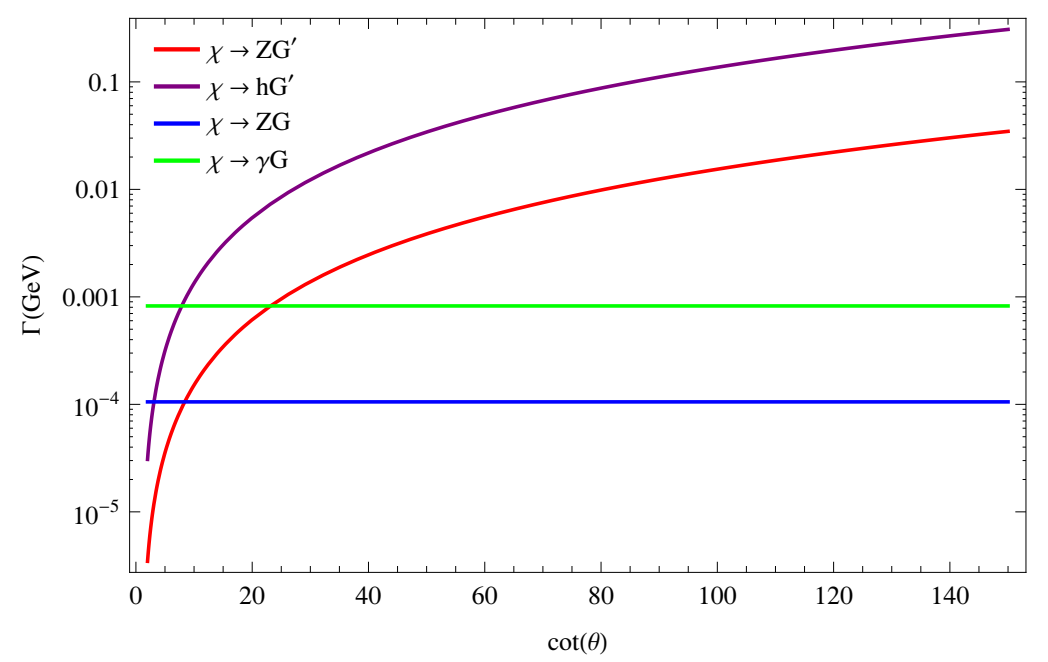

Figure 2. The partial widths of a bino-like neutralino, decaying to a pseudo-goldstino $\left(G^{\prime}\right)$ or real goldstino $(G)$, as a function of $\cot \theta$.

$\mu$ and the SM-like Higgs mass (we take $125 \mathrm{GeV}$ ) are fixed. Note that these Higgs soft parameters receive contributions from two SUSY-breaking sectors and we assume the two contributions are equal (say $B_{\mu, 1}=B_{\mu, 2}$ ) in the following numerical example.

With the above fixed parameters, we vary $\cot \theta$ and show the decay widths in figure 2 . As expected, for a small $\cot \theta$ the decays into real goldstino are important while for a large $\cot \theta$ the decays into pseudo-goldstino become dominant. The reason is obvious: the couplings of pseudo-goldstino are proportional to $\tilde{m}_{\phi}$, which can be enhanced by a large $\cot \theta$, as shown in eq. (2.6).

\section{Phenomenological study at LHC}

In this section we study the direct productions of the lightest neutralino and chargino followed by the decays to pseudo-goldstino at the LHC. In our study we assume that other SUSY particles (like squarks, sleptons, heavy Higgs bosons and gluino) are heavy enough to be decoupled. The mass of the SM-like Higgs boson is fixed at $m_{h}=125 \mathrm{GeV}$. For the parameters $M_{1}, M_{2}$ and $\mu$, they will be fixed with different values in three different cases listed in the preceding section. The sign of $\mu$ is assumed to be positive and $\tan \beta$ is fixed as 10 in the calculation. We use SOFTSUSY [30] to calculate the mass spectrum and the mixing matrices.

We use MadGraph5 [31] to perform Monte Carlo simulations for the signals and the SM backgrounds. The effective Lagrangian in eq. (2.15) for the pseudo-goldstino interaction is implemented in FeynRules [32] and passed the UFO model file [33] to MadGraph5. The signal and background samples are generated at parton level by MadGraph5 and then passed to Pythia [34] for parton shower and hadronization. The cross section of the signal is normalized to the Next-to-Leading-Order (NLO) by using Prospino2 [35]. The fast detector simulations are performed by using Delphes [36] with the ATLAS detector. For the clustering jets we use the anti- $k_{t}$ algorithm [37] with the radius parameter $\Delta R=0.5$ in the FastJet package [38]. The sample analysis is performed with the package MadAnalysis5 [39]. 


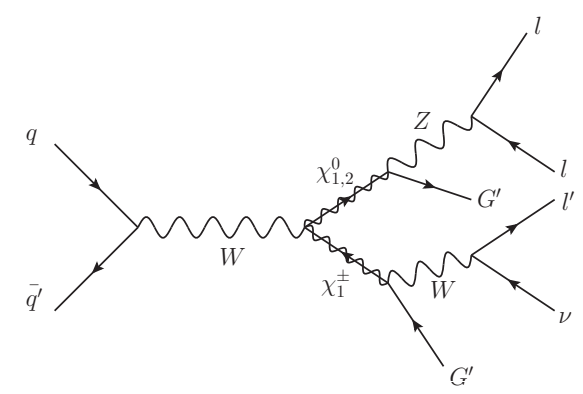

Figure 3. Feynman diagram for the pair production of $\chi_{1,2}^{0} \chi_{1}^{ \pm}$followed by the subsequent decays into pseudo-goldstino.

\subsection{Higgsino-like LOSP $\left(|\mu|<M_{1}, M_{2}\right)$}

In this case the neutralino and chargino are produced mainly through the pairs $\chi_{1}^{0} \chi_{1}^{ \pm}$, $\chi_{2}^{0} \chi_{1}^{ \pm}, \chi_{1}^{+} \chi_{1}^{-}, \chi_{1}^{0} \chi_{2}^{0}$ (Note that if $\mu$ is much smaller than $M_{1}$ and $M_{2}$, then the higgsino-like $\chi_{1}^{0}, \chi_{2}^{0}$ and $\chi_{1}^{ \pm}$are nearly degenerate and such pair productions give no visible final states in the conventional MSSM with $\chi_{1}^{0}$ being the LSP. In this case, to detect such productions at the LHC, an extra jet or photon is needed [40-42]). Their cross sections at the NLO can be found in [27]. Among these channels the production of $\chi_{1,2}^{0} \chi_{1}^{ \pm}$has the largest rate. In the two-hidden-sector SUSY breaking scenario, the neutralino decays to a $Z$-boson or Higgs plus a pseudo-goldstino $G^{\prime}$, as discussed in section II. Due to the large systematic uncertainty for the Higgs hadronic decay at the LHC, in this work we focus on the $Z$-boson mode and assume its branching ratio to be 0.5 . With the leptonic decays of $Z / W^{ \pm}$, the signal is

$$
p p \rightarrow \chi_{1,2}^{0} \chi_{1}^{ \pm} \rightarrow Z G^{\prime} W^{ \pm} G^{\prime} \rightarrow \ell^{+} \ell^{-} \ell^{ \pm} \nu G^{\prime} G^{\prime} \rightarrow 3 \ell+H_{T}, \quad(\ell=e, \mu, \tau) .
$$

The relevant Feynman diagram is displayed in figure 3. Here the three leptons in the final state contain an oppositely charged lepton pair with same flavor. The tau lepton can be partially reconstructed from its hadronic decays. Note that the neutralino pair $\chi_{1}^{0} \chi_{2}^{0}$ can also contribute to the signal. We checked that its contribution is very small and can be neglected safely. The relevant mass parameters are fixed to $\mu=200 \mathrm{GeV}, M_{1}=1.0 \mathrm{TeV}$ and $M_{2}=1.5 \mathrm{TeV}$ as a benchmark scenario in the calculation.

For the $3 \ell+E_{T}$ final state, the dominant irreducible SM background is the $W Z$ diboson production. We also consider other SM backgrounds including the top quark pair production, the di-boson production of $Z Z$, the $Z$-boson production in association with jets. The top pair production with di-leptonic decays may fake the signal since the $b$-jets and light jets may be misidentified as charged leptons. The contribution from this process can be suppressed by applying $b$-jets and light jets veto. For the background process $Z Z$ with both $Z$ bosons decaying to leptons, it can mimic our signal when one of the leptons is missing in the detector. In the case of $Z+j$ background, it may mimic our signal since a light jet may fake to charged lepton. These processes could be suppressed by requiring a large $\mathscr{H}_{T}$. We do not consider the multi-lepton $(n \geq 3)$ final state from the production of three gauge bosons due to its small cross section compared with other backgrounds. 

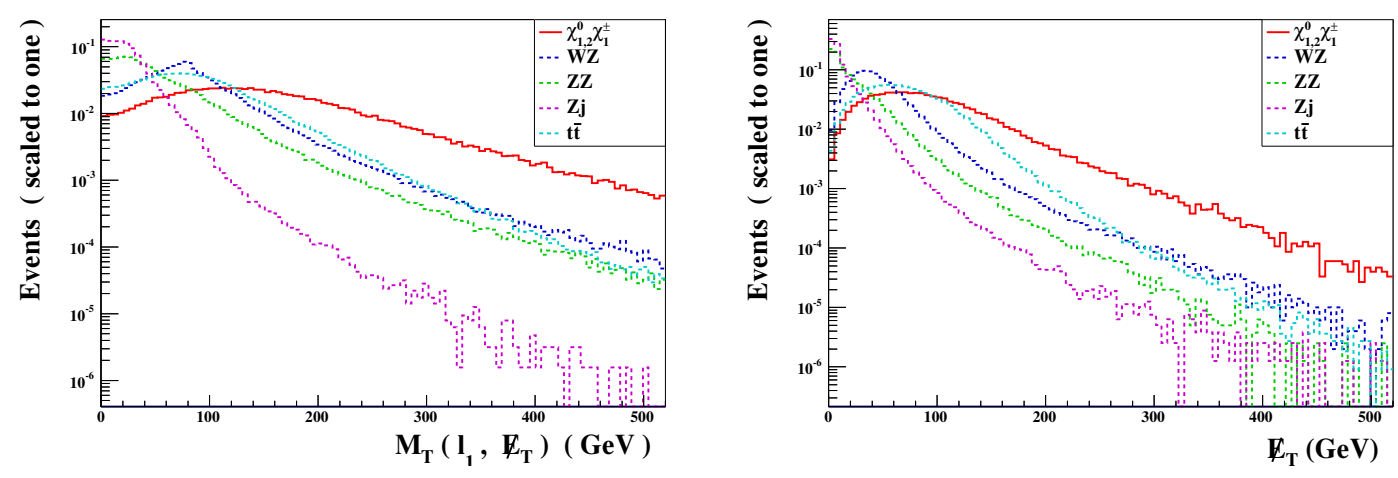

Figure 4. The normalized $M_{T}$ and $E_{T}$ distributions for the signal $p p \rightarrow \chi_{1,2}^{0} \chi_{1}^{ \pm} \rightarrow Z G^{\prime} W^{ \pm} G^{\prime} \rightarrow$ $\ell^{+} \ell^{-} \ell^{ \pm} \nu G^{\prime} G^{\prime} \rightarrow 3 \ell+E_{T}$ and background processes at the LHC with $\sqrt{s}=14 \mathrm{TeV}$. For the signal we fixed the relevant mass parameters as $\mu=200 \mathrm{GeV}, M_{1}=1.0 \mathrm{TeV}, M_{2}=1.5 \mathrm{TeV}$.

To efficiently cut the SM backgrounds, we in figure 4 plot some kinematic distributions for the signal and the backgrounds at the LHC with $\sqrt{s}=14 \mathrm{TeV}$. In the left frame of figure 4 , we give the normalized transverse mass $M_{T}\left(\ell_{1}, E_{T}\right)$ distribution, where the definition of this variable is

$$
M_{T}=\sqrt{2 p_{T}^{\ell} E_{T}\left[1-\cos \Delta \phi_{\ell, E_{T}}\right]},
$$

with $\Delta \phi_{\ell, E_{T}}$ being the azimuthal angle difference between the lepton and the missing energy. Here we use the lepton with the largest transverse momentum for constructing $M_{T}$. The right frame in figure 4 shows the normalized $\not_{T}$ distribution. It is easy to see that a lower cut of about $120 \mathrm{GeV}$ for $M_{T}$ and $100 \mathrm{GeV}$ for $\not_{T}$ can improve the statistical significance of the signal. Based on these distributions, we apply the following event selection:

- basic selection: three leptons with $p_{T}^{\ell_{1}, \ell_{2}, \ell_{3}}>60,40,20 \mathrm{GeV},|\eta|<2.5$. We use the following isolation criterion for electrons and muons: the transverse momentum sum of all charged particles with $p_{T}^{\min }>0.5 \mathrm{GeV}$ that lie within a cone $R=0.5$ around electron or muon should be less than $10 \%$ of transverse momentum of central electron or muon. Note that we assume the $\tau$-tagging efficiency to be $40 \%$ and also include the mis-tags of QCD jets in Delphes.

- $M_{T}\left(\ell_{1}, \not_{T}\right)>120 \mathrm{GeV}$.

- $E_{T}>100 \mathrm{GeV}$.

- The invariant mass of the oppositely charged lepton pair with same flavor must be within $\left|m_{\ell \ell}-m_{Z}\right|<20 \mathrm{GeV}$.

- Veto on tagged $b$-jets with $p_{T}>20 \mathrm{GeV}$ and $|\eta|<2.5$. We use the $b$-jet tagging and $c$-jet mis-tagging efficiency parametrization. ${ }^{2}$ Delphes also includes misidentification rate for light jets.

- Veto events with $p_{T}(j)>60 \mathrm{GeV}$ and $|\eta|<5.0$.

\footnotetext{
${ }^{2}$ The ATLAS parametric approach at default in Delphes.
} 


\begin{tabular}{|c|c|c|c|c|c|}
\hline cut & $W Z \rightarrow \ell \ell \ell \nu$ & $Z Z \rightarrow \ell \ell \ell \ell$ & $Z j \rightarrow \ell \ell j$ & $t \bar{t} \rightarrow b b \ell \ell \nu \nu$ & signal \\
\hline basic selection & 7240 & 540 & 17133 & 24809 & 249 \\
\hline$M_{T}\left(\ell_{1}, E_{T}\right)>120 \mathrm{GeV}$ & 2690 & 86 & 1365 & 11824 & 205 \\
\hline$E_{T}>100 \mathrm{GeV}$ & 870 & 20 & 0 & 3563 & 129 \\
\hline$\left|m_{\ell \ell}-m_{Z}\right|<20 \mathrm{GeV}$ & 834 & 18 & 0 & 568 & 123 \\
\hline veto on b-jets & 832 & 18 & 0 & 438 & 123 \\
\hline veto on light jet & 781 & 15 & 0 & 237 & 114 \\
\hline
\end{tabular}

Table 1. The numbers of events for signal $p p \rightarrow \chi_{1,2}^{0} \chi_{1}^{ \pm} \rightarrow Z G^{\prime} W^{ \pm} G^{\prime} \rightarrow \ell^{+} \ell^{-} \ell^{ \pm} \nu G^{\prime} G^{\prime} \rightarrow 3 \ell+\not_{T}$ and backgrounds at the LHC with $\sqrt{s}=14 \mathrm{TeV}$ and $100 \mathrm{fb}^{-1}$ of integrated luminosity.

\begin{tabular}{|c|c|c|c|c|c|c|}
\hline$\sqrt{s}=14 \mathrm{TeV}$ & $100 \mathrm{fb}^{-1}$ & $200 \mathrm{fb}^{-1}$ & $300 \mathrm{fb}^{-1}$ & $400 \mathrm{fb}^{-1}$ & $500 \mathrm{fb}^{-1}$ & $600 \mathrm{fb}^{-1}$ \\
\hline$S_{1 \text { [basic selection] }}$ & 249 & 498 & 747 & 996 & 1245 & 1494 \\
\hline$S_{2[\text { passing all cuts }]}$ & 114 & 228 & 342 & 456 & 570 & 684 \\
\hline$S_{1} / \sqrt{S_{1}+B_{1}}$ & 1.1 & 1.6 & 1.9 & 2.2 & 2.5 & 2.7 \\
\hline$S_{2} / \sqrt{S_{2}+B_{2}}$ & 3.4 & 4.8 & 5.8 & 6.7 & 7.5 & 8.2 \\
\hline
\end{tabular}

Table 2. The numbers of signal events for $p p \rightarrow \chi_{1,2}^{0} \chi_{1}^{ \pm} \rightarrow Z G^{\prime} W^{ \pm} G^{\prime} \rightarrow \ell^{+} \ell^{-} \ell^{ \pm} \nu G^{\prime} G^{\prime} \rightarrow 3 \ell+E_{T}$ and its statistical significance at the LHC with $\sqrt{s}=14 \mathrm{TeV}$ and different luminosities. $S_{1}$ and $B_{1}$ stand for the signal and background events after basic selection, while $S_{2}$ and $B_{2}$ stand for the signal and background events after all the cuts.

In table 1 we present the numbers of signal and background events for the LHC with $\sqrt{s}=14 \mathrm{TeV}$ and $100 \mathrm{fb}^{-1}$ of integrated luminosity. We have normalized the cross section of the $W Z$ production to NLO [43] and $t \bar{t}$ production to next-to-next-to-leading order (NNLO) [44]. From this table we can see that the signal is overwhelmed by the backgrounds after basic selection. As we excepted, the cut on the transverse mass $M_{T}$ can suppress all the background processes significantly, especially for the electroweak processes. They are further reduced by requiring large missing transverse energy. Then the dominant irreducible SM background $W Z$ is suppressed by about one order. The large background $Z j$ has been completely removed. The other important background $t \bar{t}$ is also reduced by about a factor of seven. But the signal is decreased only a half. Though the invariant mass of charged lepton pair cut $\left|m_{\ell \ell}-m_{Z}\right|<20 \mathrm{GeV}$ reduces both the signal and backgrounds, it improves the statistical significance of the signal efficiently. The final two cuts vetoing on $b$-jets and light jets are of crucial importance to further suppress the $t \bar{t}$ background. Note that the veto on the light jet also has a small effect on the signal due to the tau jet in the signal. After all cuts, the signal-to-background ratio is $11 \%$.

In table 2 we show the number of signal events and its significance before and after cuts for different luminosities at the $14 \mathrm{TeV}$ LHC. Although the signal is reduced by applying cuts, its statistical significance is increased efficiently. With an integrated luminosity of 200-300 $\mathrm{fb}^{-1}$, the sensitivity can reach $5 \sigma$. 

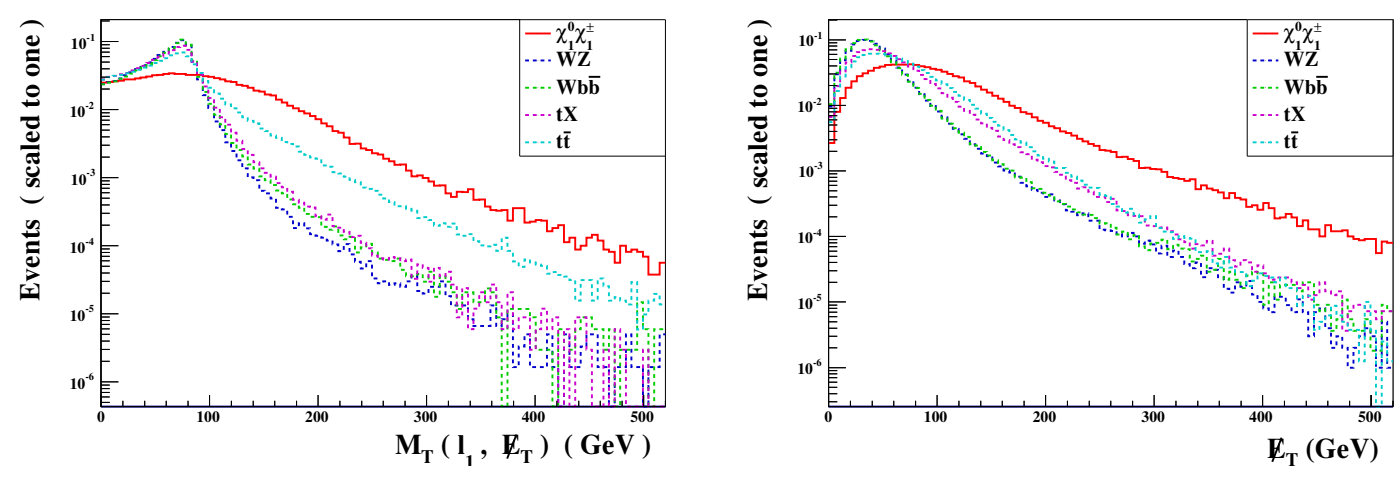

Figure 5. The normalized $M_{T}$ and $\not_{T}$ distributions for the signal $p p \rightarrow \chi_{1}^{0} \chi_{1}^{ \pm} \rightarrow h W^{ \pm} G^{\prime} G^{\prime} \rightarrow$ $\ell^{ \pm} \nu b \bar{b} G^{\prime} G^{\prime} \rightarrow \ell+2 b+\not_{T}$ and backgrounds at the LHC with $\sqrt{s}=14 \mathrm{TeV}$. For the signal we fixed the relevant mass parameter as $M_{2}=200 \mathrm{GeV}, \mu=1.0 \mathrm{TeV}, M_{1}=1.5 \mathrm{TeV}$. The other parameters are same as in figure 4.

\subsection{Wino-like LOSP $\left(M_{2}<M_{1},|\mu|\right)$}

In this case, among the direct productions of neutralinos and charginos at the LHC, the pair production of $\chi_{1}^{0} \chi_{1}^{ \pm}$is dominant and we consider this process in our analysis. As discussed before, the LOSP $\chi_{1}^{0}$ can only decay to a Higgs boson and a pseudo-goldstino $G^{\prime}$ in this case. Thus the signal is a single lepton and two bottom quarks with large missing transverse energy:

$$
p p \rightarrow \chi_{1}^{0} \chi_{1}^{ \pm} \rightarrow h W^{ \pm} G^{\prime} G^{\prime} \rightarrow \ell^{ \pm} b \bar{b} \nu G^{\prime} G^{\prime} \rightarrow \ell+2 b+\not_{T} \quad(\ell=e, \mu, \tau) .
$$

In the calculation we fix the relevant parameters as $M_{2}=200 \mathrm{GeV}, \mu=1.0 \mathrm{TeV}$ and $M_{1}=1.5 \mathrm{TeV}$. The other parameters are assumed to take the same values as in the Higgsino-like case.

The dominant SM backgrounds for this signal are di-boson productions, $W b \bar{b}$, top pair and single top productions. For di-boson productions, we only consider the $W Z$ production where $Z$ decays to $b \bar{b}$ and $W$ decays leptonically. The contribution from other di-boson productions should be very small. For the $W b \bar{b}$ production, its contribution may be suppressed by requiring large missing transverse energy. The top pair production can mimic the signal if one of the $W$ bosons decays leptonically. The single top production can also fake the signal when the light quark is misidentified as a $b$-quark or missing transverse energy.

In figure 5 we present the normalized $M_{T}$ and $E_{T}$ distributions of the signal and backgrounds at the $14 \mathrm{TeV}$ LHC. It is expected that the peak of the transverse mass distribution for the backgrounds with a single $W$ is around $m_{W}$. Including di-leptonical channels, the shape of the curves for top pair production should be a little different. We can observe that the transverse mass cut should be effective for suppressing the backgrounds. In the missing transverse energy distribution, we see that the signal has a slightly harder $E_{T}$ spectrum due to the contribution of pseudo-goldstino. Thus a hard cut on $\mathbb{E}_{T}$ will further reduce the backgrounds. At last we employ the following selections for this signal: 


\begin{tabular}{|c|c|c|c|c|c|}
\hline cut & $W Z \rightarrow \ell \nu b \bar{b}$ & $W b \bar{b} \rightarrow \ell \nu b \bar{b}$ & $t X \rightarrow \ell b \nu X$ & $t \bar{t} \rightarrow b \bar{b} \ell \nu \ell \nu\left(q q^{\prime}\right)$ & signal \\
\hline basic selection & 373 & 7845 & 50015 & 796066 & 956 \\
\hline$\left|m_{b b}-m_{h}\right|<25 \mathrm{GeV}$ & 82 & 1913 & 13164 & 199941 & 769 \\
\hline$M_{T}>100 \mathrm{GeV}$ & 4 & 220 & 1215 & 27845 & 367 \\
\hline$\not_{T}>120 \mathrm{GeV}$ & 1 & 3 & 69 & 2617 & 149 \\
\hline
\end{tabular}

Table 3. The numbers of events for signal $p p \rightarrow \chi_{1}^{0} \chi_{1}^{ \pm} \rightarrow h W^{ \pm} G^{\prime} G^{\prime} \rightarrow \ell^{ \pm} b \bar{b} \nu G^{\prime} G^{\prime} \rightarrow \ell+2 b+\not{ }_{T}$ and backgrounds for the LHC with $\sqrt{s}=14 \mathrm{TeV}$ and $100 \mathrm{fb}^{-1}$ of integrated luminosity.

\begin{tabular}{|c|c|c|c|c|c|c|}
\hline$\sqrt{s}=14 \mathrm{TeV}$ & $100 \mathrm{fb}^{-1}$ & $200 \mathrm{fb}^{-1}$ & $300 \mathrm{fb}^{-1}$ & $400 \mathrm{fb}^{-1}$ & $500 \mathrm{fb}^{-1}$ & $600 \mathrm{fb}^{-1}$ \\
\hline$S_{1 \text { [basic selection] }}$ & 956 & 1912 & 2868 & 3824 & 4780 & 5736 \\
\hline$S_{2[\text { passing all cuts] }}$ & 149 & 298 & 447 & 596 & 745 & 894 \\
\hline$S_{1} / \sqrt{S_{1}+B_{1}}$ & 1.0 & 1.5 & 1.8 & 2.1 & 2.3 & 2.5 \\
\hline$S_{2} / \sqrt{S_{2}+B_{2}}$ & 2.8 & 4.0 & 4.8 & 5.6 & 6.3 & 6.8 \\
\hline
\end{tabular}

Table 4. The number of the signal events $p p \rightarrow \chi_{1}^{0} \chi_{1}^{ \pm} \rightarrow h W^{ \pm} G^{\prime} G^{\prime} \rightarrow \ell^{ \pm} b \bar{b} \nu G^{\prime} G^{\prime} \rightarrow \ell+2 b+\not{ }_{T}$ and its statistical significance for the LHC with $\sqrt{s}=14 \mathrm{TeV}$ and different luminosities. $S_{1}$ and $B_{1}$ stand for the signal and background events after basic selection, while $S_{2}$ and $B_{2}$ stand for the signal and background events after all the cuts.

- basic selection: one isolated lepton with $p_{T}>40 \mathrm{GeV},|\eta|<2.5$ and two tagged $b$-jets with $p_{T}^{b_{1}, b_{2}}>60,40 \mathrm{GeV},|\eta|<2.5$.

- The invariant mass of $b$-jets must be within $\left|m_{b b}-m_{h}\right|<25 \mathrm{GeV}$.

- $M_{T}>100 \mathrm{GeV}$.

- $E_{T}>120 \mathrm{GeV}$.

In table 3 we display the cut flow for the signal and backgrounds at the LHC with $\sqrt{s}=14 \mathrm{TeV}$ and an integrated luminosity of $100 \mathrm{fb}^{-1}$. Note that we have normalized the dominant $t \bar{t}$ background to NNLO [44]. We see that the invariant mass cut strongly suppresses the backgrounds, while having little effect on the signal. As we have shown in figure 5 , the rather hard cuts on $M_{T}$ and $\mathscr{E}_{T}$ can efficiently reduce the SM backgrounds. We observe from table 3 that these cuts can almost remove the $W b \bar{b}$ background. The dominant top pair and single top backgrounds are also reduced by about several orders of magnitude. However, the signal is only suppressed by a factor of seven.

In table 4 we present the number of signal events and its statistical significance for different luminosities at the $14 \mathrm{TeV}$ LHC. As expected, these optimization cuts improved the signal significance efficiently. We see that the significance can reach $5 \sigma$ for an integrated luminosity of about $300 \mathrm{fb}^{-1}$. We also notice that the ratio of signal-to-background is only about $6 \%$. This implies that the systematic uncertainty must be controlled at percent level in order to detect the signal in this case. 


\subsection{Bino-like LOSP $\left(M_{1}<M_{2},|\mu|\right)$}

In this case the lightest neutralino is bino-like and its pair production cross section is small at the LHC $\left(10^{-6}-10^{-7} \mathrm{pb}\right)$. For the next lightest ordinary supersymmetric particle (NLOSP), its components depend on the relative values of $M_{2}$ and $\mu$. In the following we investigate the different scenarios: (i) $|\mu|<M_{2}$, in which the next lightest neutrilino $\chi_{2}^{0}$ and chargino $\chi_{1}^{ \pm}$are higgsino-like; (ii) $M_{2}<|\mu|$, in which the next lightest neutrilino $\chi_{2}^{0}$ and chargino $\chi_{1}^{ \pm}$are wino-like. In both scenarios, the leading production channels are the NLOSP pair production. Since the decay of the neutral NLOSP is more sensitive to the SUSY parameters than the charged NLOSP, we therefore only explore the charged NLOSP pair $\left(\chi_{1}^{+} \chi_{1}^{-}\right)$production. Here the chargino dominantly decays to a $W$ boson plus a bino-like LOSP $\chi_{1}^{0}$ or pseudo-goldstino $G^{\prime}$.

In case of a higgsino-like $\chi_{1}^{ \pm}$, due to the relative large higgsino-bino mixing, $\chi_{1}^{ \pm}$dominantly decays to $\chi_{1}^{0}$ and $W$ boson. As discussed in section II, a bino-like $\chi_{1}^{0}$ decays to Higgs and pseudo-goldstino $G^{\prime}$. Then this channel is $p p \rightarrow \chi_{1}^{+} \chi_{1}^{-} \rightarrow \chi_{1}^{0} W^{+} \chi_{1}^{0} W^{-} \rightarrow$ $h h W^{+} W^{-} G^{\prime} G^{\prime}(6.7 \mathrm{fb})$. So its cross section is too small to be detected at the LHC.

In case of a wino-like $\chi_{1}^{ \pm}$, there is little mixing between bino and wino. Then $\chi_{1}^{ \pm}$will decay to pseudo-goldstino $G^{\prime}$ and $W$ boson. Thus the signal is

$$
p p \rightarrow \chi_{1}^{+} \chi_{1}^{-} \rightarrow W^{+} G^{\prime} W^{-} G^{\prime} \rightarrow \ell^{+} \ell^{-} \nu \nu G^{\prime} G^{\prime} \rightarrow 2 \ell+E_{T} \quad(l=e, \mu, \tau) .
$$

The characteristic of this signal is two highly boosted leptons and large missing transverse energy in the final state. This feature will help to distinguish the signal from backgrounds. In our analysis the bino-like LOSP neutralino is set as $M_{1}=200 \mathrm{GeV}$. Also, we set $M_{2}=500 \mathrm{GeV}$ and $\mu=1.0 \mathrm{TeV}$, and other parameters are the same as in the higgsino-like LOSP case.

The SM backgrounds come from the di-boson productions of $W W, Z Z$ and $W Z$, the top pair and single top productions. The $W W$ background can be suppressed by requiring large missing transverse energy. For $Z Z$ background process, when one of $Z$ bosons decays to leptons and the other to neutrinos, it can resemble our signal. These two leptons are different from the signal with highly boosted leptons. Thus a high invariant mass cut on the two leptons could reduce this background. For the $W Z$ background, it will fake the signal only if one of three leptons in the final state is missing detection. The two $W$ bosons produced in $t \bar{t}$ and $t W$ processes decay to leptons and thus can fake our signal. These processes could be suppressed by applying $b$-jet and light jet vetos. Since we require large transverse energy, the $W / Z$ production associated with a jet or photon will not be considered in our work.

In figure 6 we show the normalized $M_{T}$ distributions of the hard and light charged leptons for the signal and backgrounds at the $14 \mathrm{TeV}$ LHC. Since both leptons in the signal come from the decays of heavy particles, the signal has harder spectrum than backgrounds in the $M_{T}$ distributions. We notice that the backgrounds in the $M_{T}\left(\ell_{2}, E_{T}\right)$ distribution have faster falling than in the $M_{T}\left(\ell_{1}, E_{T}\right)$ distribution. Thus we will require a cut on $M_{T}\left(\ell_{2}, \mathbb{E}_{T}\right)$ to suppress the backgrounds. The normalized $\mathscr{E}_{T}$ distribution for the signal and backgrounds is also presented in figure 6 . We see the $\mathscr{E}_{T}$ distribution for the signal is much harder than the signal due to extra pseudo-goldstino contribution to the missing energy. 

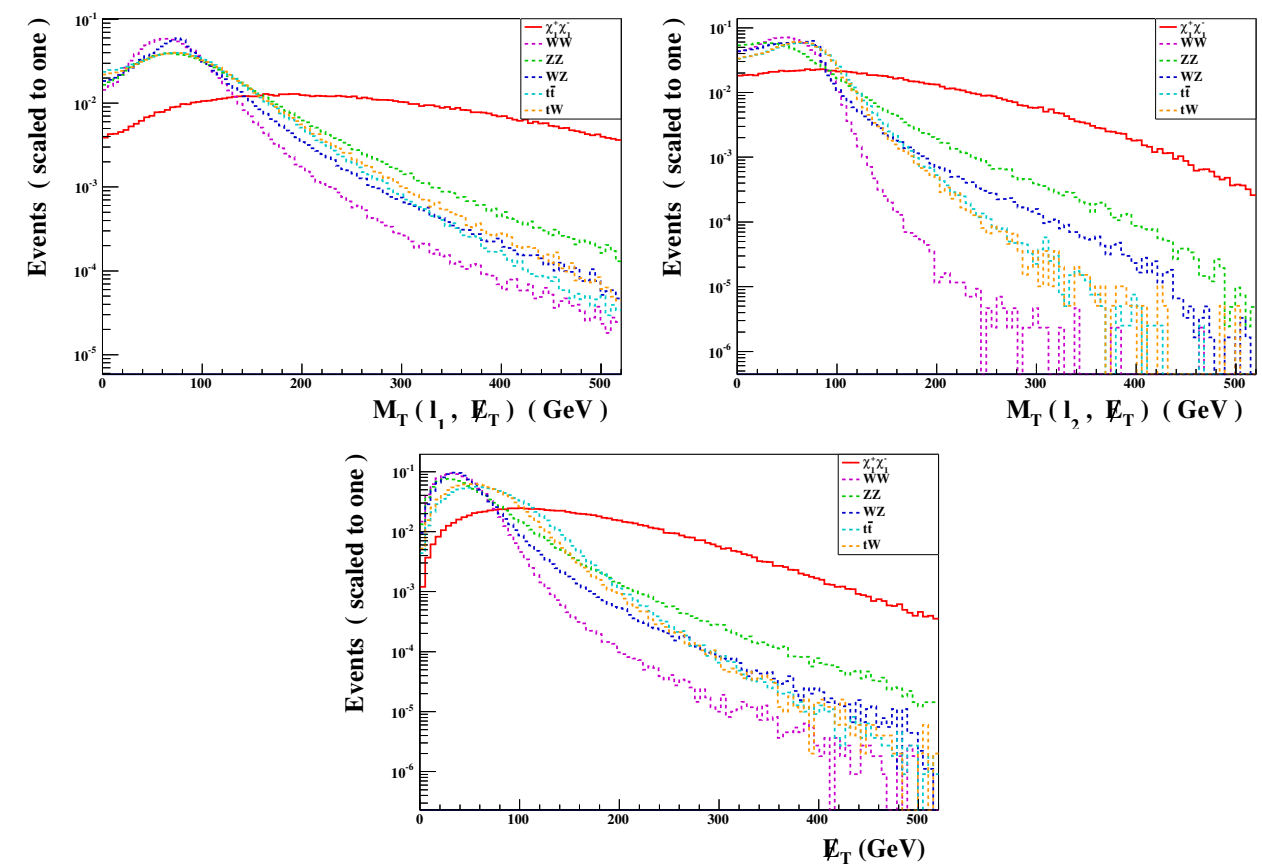

Figure 6. The normalized $M_{T}$ and $\not_{T}$ distribution for the signal $p p \rightarrow \chi_{1}^{+} \chi_{1}^{-} \rightarrow W^{+} G^{\prime} W^{-} G^{\prime} \rightarrow$ $\ell^{+} \ell^{-} \nu \nu G^{\prime} G^{\prime} \rightarrow 2 \ell+E_{T}$ and background processes at the LHC with $\sqrt{s}=14 \mathrm{TeV}$. For the signal we fixed the relevant mass parameters as $M_{1}=200 \mathrm{GeV}, M_{2}=500 \mathrm{GeV}, \mu=1.0 \mathrm{TeV}$. Other parameters are same as in figure 4 .

We will apply a large missing transverse energy cut to improve the signal significance. Based on the above analysis, we apply the following selection for this signal:

- basic selection: two opposite-sign leptons with $P_{T}^{\ell_{1}, \ell_{2}}>60,40 \mathrm{GeV},|\eta|<2.5$.

- $M_{T}\left(\ell_{2}, \not_{T}\right)>120 \mathrm{GeV}$.

- $H_{T}>120 \mathrm{GeV}$.

- $M_{\ell^{+} \ell^{-}}>140 \mathrm{GeV}$.

- Veto on tagged $b$-jets with $P_{T}>20 \mathrm{GeV}$ and $|\eta|<2.5$.

- Veto events with $P_{T}(j)>50 \mathrm{GeV}$ and $|\eta|<5.0$.

In table 5 we present the cut flow for the signal and background events at the LHC with $\sqrt{s}=14 \mathrm{TeV}$ and an integrated luminosity of $100 \mathrm{fb}^{-1}$. We have normalized the dominant $t \bar{t}$ background to NNLO [44]. We see that the signal is overwhelmed by the backgrounds at the basic selection level. As we expected, the $M_{T}$ cut on the light lepton can suppress the backgrounds, while keeping most of the signal. This cut is extremely effective for suppressing the $W W$ background. Then the $W W$ background is further suppressed by a hard cut on $\not_{T}$. The $W Z$ and $Z Z$ backgrounds with two leptons from $Z$ decay are removed by requiring a large invariant mass of leptons. The dominant reducible backgrounds $t \bar{t}$ and $t W$ are suppressed strongly by the veto on $b$-jets and light jets. After all cuts, the signalto-background ratio is about $25 \%$. 


\begin{tabular}{|c|c|c|c|c|c|c|}
\hline cut & $W W \rightarrow \ell \ell \nu \nu$ & $Z Z \rightarrow \ell \ell \nu \nu$ & $W Z \rightarrow \ell \ell \ell \nu$ & $t \bar{t} \rightarrow b \bar{b} \ell \ell \nu \nu$ & $t W \rightarrow b \ell \ell \nu \nu$ & signal \\
\hline basic selection & 30524 & 1524 & 1578 & 599505 & 52913 & 102 \\
\hline$M_{T}\left(\ell_{2}, E_{T}\right)>120 \mathrm{GeV}$ & 744 & 900 & 407 & 84647 & 6018 & 65.7 \\
\hline$E_{T}>120 \mathrm{GeV}$ & 12.6 & 582 & 180 & 14381 & 901 & 55.5 \\
\hline$M_{\ell^{+} \ell^{-}}>140 \mathrm{GeV}$ & 11.4 & 0.4 & 5.3 & 9759 & 643 & 43.3 \\
\hline veto on b-jets & 11.1 & 0.4 & 5.3 & 4107 & 334 & 43.1 \\
\hline veto on light jet & 6.1 & 0.3 & 1.9 & 124 & 17.9 & 37.3 \\
\hline
\end{tabular}

Table 5. The numbers of events for signal $p p \rightarrow \chi_{1}^{+} \chi_{1}^{-} \rightarrow W^{+} G^{\prime} W^{-} G^{\prime} \rightarrow \ell^{+} \ell^{-} \nu \nu G^{\prime} G^{\prime} \rightarrow 2 \ell+\not \ell_{T}$ and background at the LHC with $\sqrt{s}=14 \mathrm{TeV}$ and $100 \mathrm{fb}^{-1}$ of integrated luminosity.

\begin{tabular}{|c|c|c|c|c|c|c|}
\hline$\sqrt{s}=14 \mathrm{TeV}$ & $100 \mathrm{fb}^{-1}$ & $200 \mathrm{fb}^{-1}$ & $300 \mathrm{fb}^{-1}$ & $400 \mathrm{fb}^{-1}$ & $500 \mathrm{fb}^{-1}$ & $600 \mathrm{fb}^{-1}$ \\
\hline$S_{1 \text { [basic selection] }}$ & 102 & 204 & 306 & 408 & 510 & 612 \\
\hline$S_{2[\text { passing all cuts }]}$ & 37.3 & 74.6 & 112 & 149 & 187 & 224 \\
\hline$S_{1} / \sqrt{S_{1}+B_{1}}$ & 0.12 & 0.17 & 0.21 & 0.25 & 0.28 & 0.30 \\
\hline$S_{2} / \sqrt{S_{2}+B_{2}}$ & 2.7 & 3.9 & 4.7 & 5.4 & 6.1 & 6.8 \\
\hline
\end{tabular}

Table 6. The number of events for the signal $p p \rightarrow \chi_{1}^{+} \chi_{1}^{-} \rightarrow W^{+} G^{\prime} W^{-} G^{\prime} \rightarrow \ell^{+} \ell^{-} \nu \nu G^{\prime} G^{\prime} \rightarrow$ $2 \ell+E_{T}$ and its statistical significance for the LHC with $\sqrt{s}=14 \mathrm{TeV}$ and different luminosities. $S_{1}$ and $B_{1}$ stand for the signal and background events after basic selection, while $S_{2}$ and $B_{2}$ stand for the signal and background events after all the cuts.

\begin{tabular}{|c|c|c|c|c|}
\hline$\sqrt{s}=8 \mathrm{TeV} \mathcal{L}=21 \mathrm{fb}^{-1}$ & $S$ & $B$ & $S / B$ & $S / \sqrt{S+B}$ \\
\hline Higgsino-like LOSPs & 11.2 & 90.1 & 0.12 & 1.11 \\
\hline Wino-like LOSPs & 13.4 & 169.4 & 0.08 & 0.99 \\
\hline Bino-like LOSPs & 2.0 & 10.5 & 0.19 & 0.55 \\
\hline
\end{tabular}

Table 7. The statistical significances for three different cases at the LHC with $\sqrt{s}=8 \mathrm{TeV}$ and $21 \mathrm{fb}^{-1}$.

In table 6 we display the number of signal events and its significance before and after the cuts for different luminosities at the $14 \mathrm{TeV}$ LHC. We see that the significance is improved by these cuts efficiently. The significance can reach $5 \sigma$ for a luminosity of $300-400 \mathrm{fb}^{-1}$.

Finally, we note that the LHC searched the neutralinos and charginos with leptons plus missing $E_{T}$ at 7 and $8 \mathrm{TeV}$, and the observed events are in agreement with the SM backgrounds (no excess), which gave some limits on the relevant parameter space [45-47]. Since in our scenario the signals are quite rare compared with the huge SM backgrounds (as shown in our results, only at $14 \mathrm{TeV}$ LHC with a rather high luminosity can our signals be possibly accessible), the current LHC limits at 7 and $8 \mathrm{TeV}$ with rather limited luminosities are not yet able to constrain the scenario under our consideration. We numerically checked this and the results for the $8 \mathrm{TeV}$ LHC are shown in table 7 (since the kinetic distributions of the signals and backgrounds for the $8 \mathrm{TeV}$ LHC are similar to the results for the $14 \mathrm{TeV}$ LHC, we use the same cuts as for the $14 \mathrm{TeV}$ LHC in each case). We see that the statistical significances are below $2 \sigma$ for a luminosity of $21 \mathrm{fb}^{-1}$. 


\section{Conclusion}

Pseudo-goldstino is predicted in the multi-sector SUSY breaking scenario. Comparing to the ordinary gravitino, it can couple to the visible sector more strongly and hence lead to some intriguing phenomenology at colliders. In this scenario the lightest neutralino (chargino) can decay into a pseudo-goldstino plus a $Z$-boson or Higgs boson ( $W$-boson). In this work we performed a Monte Carlo simulation for the direct productions of the lightest neutralino and chargino followed by the decays to pseudo-goldstino. Considering a higgsinolike, bino-like or wino-like lightest neutralino, we found that the signal-to-background ratio $(S / B)$ is $6 \%-25 \%$ and the statistical significance $S / \sqrt{S+B}$ is $5 \sigma$ at the high luminosity LHC. So it is feasible to explore such a multi-sector SUSY breaking scenario at the high luminosity LHC if the background is known to percent level.

\section{Acknowledgments}

Lin Wang acknowledges Prof. Johann H. Kühn and Prof. Matthias Steinhauser for their warm hospitality. This work is supported by by the Grant-in-Aid for Scientific Research (No. 24540246) from Ministry of Education, Culture, Sports, Science and Technology (MEXT) of Japan, by DFG through SFB/TR 9 "Computational Particle Physics" and by the National Natural Science Foundation of China under grant Nos. 11275245, 10821504 and 11135003.

Open Access. This article is distributed under the terms of the Creative Commons Attribution License (CC-BY 4.0), which permits any use, distribution and reproduction in any medium, provided the original author(s) and source are credited.

\section{References}

[1] M. Carena, S. Gori, N.R. Shah and C.E.M. Wagner, A $125 \mathrm{GeV}$ SM-like Higgs in the MSSM and the $\gamma \gamma$ rate, JHEP 03 (2012) 014 [arXiv:1112.3336] [INSPIRE].

[2] M. Carena, S. Gori, N.R. Shah, C.E.M. Wagner and L.-T. Wang, Light Stau Phenomenology and the Higgs $\gamma \gamma$ Rate, JHEP 07 (2012) 175 [arXiv: 1205.5842] [INSPIRE].

[3] J.-J. Cao, Z.-X. Heng, J.M. Yang, Y.-M. Zhang and J.-Y. Zhu, A SM-like Higgs near $125 \mathrm{GeV}$ in low energy SUSY: a comparative study for MSSM and NMSSM, JHEP 03 (2012) 086 [arXiv:1202.5821] [INSPIRE].

[4] J. Cao, Z. Heng, J.M. Yang and J. Zhu, Status of low energy SUSY models confronted with the LHC $125 \mathrm{GeV}$ Higgs data, JHEP 10 (2012) 079 [arXiv: 1207.3698] [INSPIRE].

[5] J. Cao, Z. Heng, D. Li and J.M. Yang, Current experimental constraints on the lightest Higgs boson mass in the constrained MSSM, Phys. Lett. B 710 (2012) 665 [arXiv:1112.4391] [INSPIRE].

[6] U. Ellwanger, A Higgs boson near $125 \mathrm{GeV}$ with enhanced di-photon signal in the NMSSM, JHEP 03 (2012) 044 [arXiv: 1112.3548] [INSPIRE].

[7] U. Ellwanger and C. Hugonie, Higgs bosons near $125 \mathrm{GeV}$ in the NMSSM with constraints at the GUT scale, Adv. High Energy Phys. 2012 (2012) 625389 [arXiv:1203.5048] [INSPIRE]. 
[8] C. Cheung, Y. Nomura and J. Thaler, Goldstini, JHEP 03 (2010) 073 [arXiv:1002.1967] [INSPIRE].

[9] C. Cheung, J. Mardon, Y. Nomura and J. Thaler, A Definitive Signal of Multiple Supersymmetry Breaking, JHEP 07 (2010) 035 [arXiv: 1004.4637] [INSPIRE].

[10] K. Benakli and C. Moura, Brane-Worlds Pseudo-Goldstinos, Nucl. Phys. B 791 (2008) 125 [arXiv:0706.3127] [INSPIRE].

[11] N. Craig, J. March-Russell and M. McCullough, The Goldstini Variations, JHEP 10 (2010) 095 [arXiv: 1007.1239] [INSPIRE].

[12] M. McCullough, Stimulated Supersymmetry Breaking, Phys. Rev. D 82 (2010) 115016 [arXiv: 1010.3203] [INSPIRE].

[13] K.I. Izawa, Y. Nakai and T. Shimomura, Higgs Portal to Visible Supersymmetry Breaking, JHEP 03 (2011) 007 [arXiv: 1101.4633] [INSPIRE].

[14] J. Thaler and Z. Thomas, Goldstini Can Give the Higgs a Boost, JHEP 07 (2011) 060 [arXiv: 1103.1631] [INSPIRE].

[15] C. Cheung, F. D'Eramo and J. Thaler, The Spectrum of Goldstini and Modulini, JHEP 08 (2011) 115 [arXiv:1104.2600] [INSPIRE].

[16] D. Bertolini, K. Rehermann and J. Thaler, Visible Supersymmetry Breaking and an Invisible Higgs, JHEP 04 (2012) 130 [arXiv:1111.0628] [INSPIRE].

[17] H.-C. Cheng, W.-C. Huang, I. Low and A. Menon, Goldstini as the decaying dark matter, JHEP 03 (2011) 019 [arXiv: 1012.5300] [INSPIRE].

[18] K. Mawatari and Y. Takaesu, HELAS and MadGraph with goldstinos, Eur. Phys. J. C 71 (2011) 1640 [arXiv:1101.1289] [inSPIRE].

[19] R. Argurio, Z. Komargodski and A. Mariotti, Pseudo-Goldstini in Field Theory, Phys. Rev. Lett. 107 (2011) 061601 [arXiv:1102.2386] [INSPIRE].

[20] R. Argurio, K. De Causmaecker, G. Ferretti, A. Mariotti, K. Mawatari and and Y. Takaesu, Collider signatures of goldstini in gauge mediation, JHEP 06 (2012) 096 [arXiv:1112.5058] [INSPIRE].

[21] G. Ferretti, A. Mariotti, K. Mawatari and C. Petersson, Multiphoton signatures of goldstini at the LHC, JHEP 04 (2014) 126 [arXiv:1312.1698] [INSPIRE].

[22] T. Liu, L. Wang and J.M. Yang, Higgs decay to goldstini and its observability at the LHC, Phys. Lett. B 726 (2013) 228 [arXiv:1301.5479] [INSPIRE].

[23] S. Samuel and J. Wess, A Superfield Formulation of the Nonlinear Realization of Supersymmetry and Its Coupling to Supergravity, Nucl. Phys. B 221 (1983) 153 [InSPIRE].

[24] R. Casalbuoni, S. De Curtis, D. Dominici, F. Feruglio and R. Gatto, Nonlinear Realization of Supersymmetry Algebra From Supersymmetric Constraint, Phys. Lett. B 220 (1989) 569 [INSPIRE].

[25] Z. Komargodski and N. Seiberg, From Linear SUSY to Constrained Superfields, JHEP 09 (2009) 066 [arXiv:0907.2441] [INSPIRE].

[26] Z. Komargodski and D. Shih, Notes on SUSY and R-Symmetry Breaking in Wess-Zumino Models, JHEP 04 (2009) 093 [arXiv: 0902.0030] [INSPIRE]. 
[27] T. Han, S. Padhi and S. Su, Electroweakinos in the Light of the Higgs Boson, Phys. Rev. D 88 (2013) 115010 [arXiv:1309.5966] [INSPIRE].

[28] S. Ambrosanio, G.L. Kane, G.D. Kribs, S.P. Martin and S. Mrenna, Search for supersymmetry with a light gravitino at the Fermilab Tevatron and CERN LEP colliders, Phys. Rev. D 54 (1996) 5395 [hep-ph/9605398] [INSPIRE].

[29] S. Dimopoulos, S.D. Thomas and J.D. Wells, Sparticle spectroscopy and electroweak symmetry breaking with gauge mediated supersymmetry breaking, Nucl. Phys. B 488 (1997) 39 [hep-ph/9609434] [InSPIRE].

[30] B.C. Allanach, SOFTSUSY: a program for calculating supersymmetric spectra, Comput. Phys. Commun. 143 (2002) 305 [hep-ph/0104145] [INSPIRE].

[31] J. Alwall, M. Herquet, F. Maltoni, O. Mattelaer and T. Stelzer, MadGraph 5: Going Beyond, JHEP 06 (2011) 128 [arXiv:1106.0522] [INSPIRE].

[32] A. Alloul, N.D. Christensen, C. Degrande, C. Duhr and B. Fuks, FeynRules 2.0 - A complete toolbox for tree-level phenomenology, Comput. Phys. Commun. 185 (2014) 2250 [arXiv: 1310.1921] [INSPIRE].

[33] C. Degrande et al., UFO - The Universal FeynRules Output, Comput. Phys. Commun. 183 (2012) 1201 [arXiv:1108.2040] [INSPIRE].

[34] T. Sjöstrand, L. Lönnblad, S. Mrenna and P.Z. Skands, PYTHIA 6.3 physics and manual, hep-ph/0308153 [INSPIRE].

[35] W. Beenakker et al., The production of charginos/neutralinos and sleptons at hadron colliders, Phys. Rev. Lett. 83 (1999) 3780 [Erratum ibid. 100 (2008) 029901] [hep-ph/9906298] [INSPIRE].

[36] DELPHES 3 collaboration, J. de Favereau et al., DELPHES 3, A modular framework for fast simulation of a generic collider experiment, JHEP 02 (2014) 057 [arXiv: 1307.6346] [INSPIRE].

[37] M. Cacciari, G.P. Salam and G. Soyez, The Anti-k(t) jet clustering algorithm, JHEP 04 (2008) 063 [arXiv:0802.1189] [INSPIRE].

[38] M. Cacciari, G.P. Salam and G. Soyez, FastJet User Manual, Eur. Phys. J. C 72 (2012) 1896 [arXiv:1111.6097] [INSPIRE].

[39] E. Conte, B. Fuks and G. Serret, MadAnalysis 5, A User-Friendly Framework for Collider Phenomenology, Comput. Phys. Commun. 184 (2013) 222 [arXiv:1206.1599] [InSPIRE].

[40] C. Han et al., Probing Light Higgsinos in Natural SUSY from Monojet Signals at the LHC, JHEP 02 (2014) 049 [arXiv: 1310.4274] [INSPIRE].

[41] P. Schwaller and J. Zurita, Compressed electroweakino spectra at the LHC, JHEP 03 (2014) 060 [arXiv: 1312.7350] [INSPIRE].

[42] H. Baer, A. Mustafayev and X. Tata, Monojets and mono-photons from light higgsino pair production at LHC14, Phys. Rev. D 89 (2014) 055007 [arXiv:1401.1162] [INSPIRE].

[43] J.M. Campbell, R.K. Ellis and C. Williams, Vector boson pair production at the LHC, JHEP 07 (2011) 018 [arXiv:1105.0020] [INSPIRE].

[44] M. Czakon, P. Fiedler and A. Mitov, Total Top-Quark Pair-Production Cross section at Hadron Colliders Through $O\left(\alpha_{S}^{4}\right)$, Phys. Rev. Lett. 110 (2013) 252004 [arXiv:1303.6254] [INSPIRE]. 
[45] CMS collaboration, Searches for electroweak production of charginos, neutralinos and sleptons decaying to leptons and $W, Z$ and Higgs bosons in pp collisions at 8 TeV, CMS-SUS-13-006 (2014).

[46] ATLAS collaboration, Search for direct production of charginos, neutralinos and sleptons in final states with two leptons and missing transverse momentum in pp collisions at $\sqrt{s}=8 \mathrm{TeV}$ with the ATLAS detector, JHEP 05 (2014) 071 [arXiv: 1403.5294] [INSPIRE].

[47] ATLAS collaboration, Search for direct production of charginos and neutralinos in events with three leptons and missing transverse momentum in $\sqrt{s}=8 \mathrm{TeV} p$ collisions with the ATLAS detector, JHEP 04 (2014) 169 [arXiv:1402.7029] [INSPIRE]. 